\title{
Development and Testing of New Screening Method for Keratan Sulfate in Mucopolysaccharidosis IVA
}

SHUNJI TOMATSU, KAZUO OKAMURA, TAKESHI TAKETANI, KOJI O. ORII, TATSUO NISHIOKA, MONICA A. GUTIERREZ, SUSANA VELEZ-CASTRILLON, ANGELA A. FACHEL, JEFFREY H. GRUBB, ALAN COOPER, MARGARET THORNLEY, ED WRAITH, LUIS A. BARRERA, ROBERTO GIUGLIANI, IDA V. SCHWARTZ, GUDRUN SCHULZE FRENKING, MICHAEL BECK, SUSANNE G. KIRCHER, EDUARD PASCHKE, SEIJI YAMAGUCHI, KURT ULLRICH, KOJI ISOGAI, YASUYUKI SUZUKI, TADAO ORII, NAOMI KONDO, MICHAEL CREER, AND AKIHIKO NOGUCHI

Edward A. Doisy Department of Biochemistry and Molecular Biology, Saint Louis University School of Medicine [S.T., K.O.O., M.A.G., S.V.-C., A.A.F., J.H.G.], Department of Pediatrics, Cardinal Glennon Children's Hospital [A.N.], Department of Pathology [M.C.], Saint Louis University, St. Louis, Missouri 63104, U.S.A., Department of Pediatrics, Shimane Medical University, Izumo Shimane-ken 693-8501, Japan [S.T., T.T., S.Y.], Seikagaku Corporation, Central Research Laboratories, Tokyo 207-0021, Japan [K.O.], Department of Pediatrics, Gifu University School of Medicine, Gifu 500, Japan [K.O.O., K.I., Y.S., T.O., N.K.], Institute of Inborn Errors of Metabolism, Javeriana University, Bogotá, Colombia [M.A.G., L.A.B.], Willink Biochemical Genetics Unit, Royal Manchester Children's Hospital, Manchester M27 4HA, U.K. [A.C., M.T., E.W.], Medical Genetics Service, Federal University of Rio Grande do Sul de Clinicas de Porto Alegre Rua Ramiro Barcelos, 2350 $90035-903$ Porto Alegre-RS, Brazil [R.G., I.V.S.], Department of Pediatrics, University of Mainz, Mainz 55130, Germany [G.S.F., M.B.], Institute of Medical Chemistry, University of Vienna, A-1090 Vienna, Austria [S.G.K.], Laboratory of Metabolic Diseases, Department of Pediatrics, University of Graz, A-8036 Graz, Austria [E.P.], and Department of Pediatrics, University of Hamburg, Hamburg 20251 Germany [K.U.]

\section{ABSTRACT}

\begin{abstract}
Mucopolysaccharidosis IVA (MPS IVA), a progressive lysosomal storage disease, causes skeletal dysplasia through excessive storage of keratan sulfate $(\mathrm{KS})$. We developed an ELISA-sandwich assay that used a MAb specific to KS. Forty-five blood and 59 urine specimens from MPS IVA patients (ages 1-65 y) were analyzed to determine whether KS concentration is a suitable marker for early diagnosis and longitudinal assessment of disease severity. Blood specimens were obtained from patients categorized as phenotypically severe $(n=36)$ and milder $(n=9)$. Urine specimens were also analyzed from patients categorized as severe $(n=56)$ and milder $(n=12)$, respectively. Blood KS levels (101-1525 ng/mL) in MPS IVA patients were two to eight times higher than those in age-matched controls (15-323 ng/mL). It was found that blood KS level varied with age and clinical severity. Blood KS levels in both MPS IVA and controls peaked between 5 and $10 \mathrm{y}$ of age (mean, 776 versus $234 \mathrm{ng} / \mathrm{mL}$, respectively). Blood levels in severe MPS IVA were 1.5 times higher than in the milder form. In contrast to blood, urine KS levels in both MPS IVA and controls peaked between 1 and 5 y (15.3 versus $0.26 \mathrm{mg} / \mathrm{g}$ creatinine),
\end{abstract}

and thereafter declined with age. Urine KS level also varied with age and clinical severity, and the severe MPS IVA phenotype was associated with 6.7 times greater urine $\mathrm{KS}$ excretion than the milder one. These findings indicate that the new assay for blood or urine KS may be suitable for early diagnosis and longitudinal assessment of disease severity in MPS IVA. (Pediatr Res 55: 592-597, 2004)

Abbreviations
CS, chondroitin sulfate
DMB, dimethylmethylene blue
DS, dermatan sulfate
GAG, glycosaminoglycans
GALNS, $N$-acetylgalactosamine-6-sulfate sulfatase
HS, heparan sulfate
KS, keratan sulfate
LSD, lysosomal storage disease
MPS, mucopolysaccharidoses
MPS IVA, mucopolysaccharidosis IVA

MPS are a family of heritable disorders caused by deficiency of lysosomal enzymes required for degradation of GAG (1). Each known MPS type involves deficiency of 1 of 11 lysoso-

Received March 7, 2003; accepted November 11, 2003.

Correspondence: S. Tomatsu, M.D., E.A. Doisy Department of Biochemistry and Molecular Biology, St. Louis University School of Medicine, 1402 S. Grand Blvd., St. Louis, MO 63104, U.S.A.; e-mail: tomatsus@slu.edu

Supported by the grants from the Austrian Research Society for MPS, the German MPS Society, the International Morquio Organization, and the Seikagaku Corporation.

DOI: 10.1203/01.PDR.0000113767.60140.E9 mal enzymes required for the stepwise degradation of DS, HS, $\mathrm{KS}$, or CS or a combination of GAG.

MPS IVA (or Morquio A syndrome) is an autosomal recessive disease caused by the deficiency of GALNS. GALNS is required to degrade KS and chondroitin-6-sulfate (C6S). The cardinal feature of MPS IVA, bone dysplasia, determines the disease severity. Clinical phenotypes of MPS IVA patients vary from the classical or severe form, characterized by bone dysplasia such as short trunk dwarfism, kyphoscoliosis, coxa valga, odontoid hypoplasia, abnormal 
gait, joint mobility problems, restriction of chest wall movement, and a lifespan of $20-30 \mathrm{y}$, to the milder form where patients can have a near normal quality of life with mild involvement of the skeleton (2).

The pathogenesis of the bone dysplasia is largely unknown, but it is believed that the accumulation of KS could be toxic to osteoblasts (3). An easily measurable blood or urine marker for the disease would be useful for making an early diagnosis, determining disease severity, and systematically monitoring patients' responses to treatment regimens.

One potential disease marker for MPS IVA, total GAG in the urine, can be measured spectrometrically using DMB (4) or Alcian blue (5). However, these methods are not applicable to blood without prior protease treatment, as protein in the specimen interferes with the binding of the dye to the GAG. In addition, the dye itself is also prone to decompose, leading to a high background. Urine total GAG levels in patients with MPS IVA are close to the normal range, so it is difficult to distinguish this disease based on urine GAG excretion (6).

Another potential disease marker could be KS levels. Although the excessive storage of KS is known to cause severe skeletal dysplasia in MPS IVA patients, no data on the levels of KS in the blood of these patients have been reported. The conventional MAb assay (ELISA inhibition) for KS measurement (7) involves multiple laborious steps. In the present study, we report a new ELISA method for detection of KS in biologic fluids. The results obtained by using this assay demonstrate that KS levels in the blood and urine of MPS IVA patients are significantly elevated compared with normal controls of equivalent ages. There was also a direct correlation between KS levels and clinical severity in MPS IVA patients.

\section{MATERIALS AND METHODS}

Subjects. Blood samples were obtained from 45 MPS IVA patients (35 severe form, 9 milder form) and urine samples were obtained from 59 MPS IVA patients ( 47 severe form, 12 milder form) after informed consent was obtained for each patient. For 48 blood (plasma) and 70 urine samples, the ages of MPS IVA patients were identified. Blood (plasma) and urine samples were also obtained from 117 and 95 normal controls, respectively. The diagnosis of MPS IVA was made based upon a reduced enzyme activity (GALNS) of $\leq 5 \%$ normal level in plasma, leukocytes, or fibroblasts. Forty-one MPS IVA patients provided both blood and urinary samples.

Materials. The KS standard samples for ELISA calibration and the anti-keratan MAb (5-D-4) (8) were obtained from Seikagaku Corp. (Tokyo, Japan). Antibody precoated plates were made by adding $50 \mu \mathrm{L}$ of $20 \mu \mathrm{g} / \mathrm{mL}$ antibody solution to each well of the microtiter plate (Maxisorp \#468667, NUNC A/S, Roskilde, Denmark).

The substrate in the last step of the ELISA was 3,3', 5,5'tetramethylbenzidine (TMB) purchased from Moss Inc. (Pasadena, MD, U.S.A.). The HS, heparin (Hep), CS-A, CS-C, and DS were obtained from Seikagaku in an acid mucopolysaccharide (AMPS) kit (\#400610). All reagents described for the ELISA are commercially available as the KS-ELISA kit
(\#280565) from Seikagaku or Associates of Cape Cod, Inc. (Falmouth, MA, U.S.A.).

\section{ELISA}

The 5-D-4 antibody was biotinylated using NHS-Biotin (\#20217) from Pierce Chemical (Rockford IL, U.S.A.). The plate-washing buffer (PBS-0.05\% Tween 20) and sample diluent (PBS containing 1\% BSA) were brought to room temperature before use. The washing buffer $(200 \mu \mathrm{L} /$ well $)$ was added to the microplate by multi-pipette then discarded. This washing procedure was repeated three times. Samples (50 $\mu \mathrm{L} /$ well) of KS standards and diluted unknown samples were added to the well and incubated at $37^{\circ} \mathrm{C}$ for $60 \mathrm{~min}$. The plate wells were then washed four times with the washing buffer. Next, $25 \mu \mathrm{L} /$ well of horseradish peroxidase conjugated streptavidin and $25 \mu \mathrm{L} /$ well of biotinylated antibody were added to the plate and incubated for $60 \mathrm{~min}$ at $37^{\circ} \mathrm{C}$. After washing the plate four times, $50 \mu \mathrm{L} /$ well of substrate solution (TMB) were added to the plate and incubated for $10 \mathrm{~min}$ at room temperature. The reaction was then stopped with $50 \mu \mathrm{L} 1 \mathrm{~N} \mathrm{HCl}$. The absorbance was measured at $450 \mathrm{~nm}$ with a microplate spectrophotometer. The KS concentration was read by applying the absorbances of each sample to the calibration curve.

\section{ANALYSIS OF URINARY TOTAL GAG}

GAG was quantitatively measured using DMB (4). Creatinine was measured by mixing $10 \mu \mathrm{L}$ of a 10 -fold diluted urine sample with $50 \mu \mathrm{L}$ saturated picric acid (Sigma Chemical, St. Louis, MO, U.S.A.) and $50 \mu \mathrm{L} 0.2 \mathrm{M} \mathrm{NaOH}$. Absorbance at $490 \mathrm{~nm}$ was read after $20 \mathrm{~min}$ and compared with the standard. The GAG/creatinine ratio (milligrams of GAG per gram of urinary creatinine) was used as a measure of the urinary excretion of GAG.

\section{DATA ANALYSIS}

The data obtained were analyzed to determine whether the levels of KS varied significantly with respect to age and clinical phenotype of MPS IVA patients. Variability of KS levels in blood and urine in both MPS IVA patient and controls were plotted according to age. For the comparison of patient versus control samples, the $t$ test, Mann-Whitney $U$ test, or Welch's $t$ test was applied depending on the distribution of the values. Correlation between blood and urine KS was examined using Pearson's correlation coefficient. A regression analysis was used to examine correlation between urine KS and total GAG. Descriptive statistics for all variables were tabulated. All data analyses were performed using StatView statistical software (Abacus Concepts, Berkeley, CA, U.S.A.).

\section{RESULTS}

Assay validation. The precision, expressed as the coefficient of variation $(\mathrm{CV} \%)$, was calculated on repeated measurements of KS in plasma and urinary samples performed on the same day. We found that optimized KS calibration curves gave a good standard curve $\left(R^{2}=0.9999\right)$ (Fig. 1) between $2.5 \mathrm{ng} / \mathrm{mL}$ and $40 \mathrm{ng} / \mathrm{mL}$. The intra-assay CV at 2.5, 5.0, 10.0, 20.0, and 


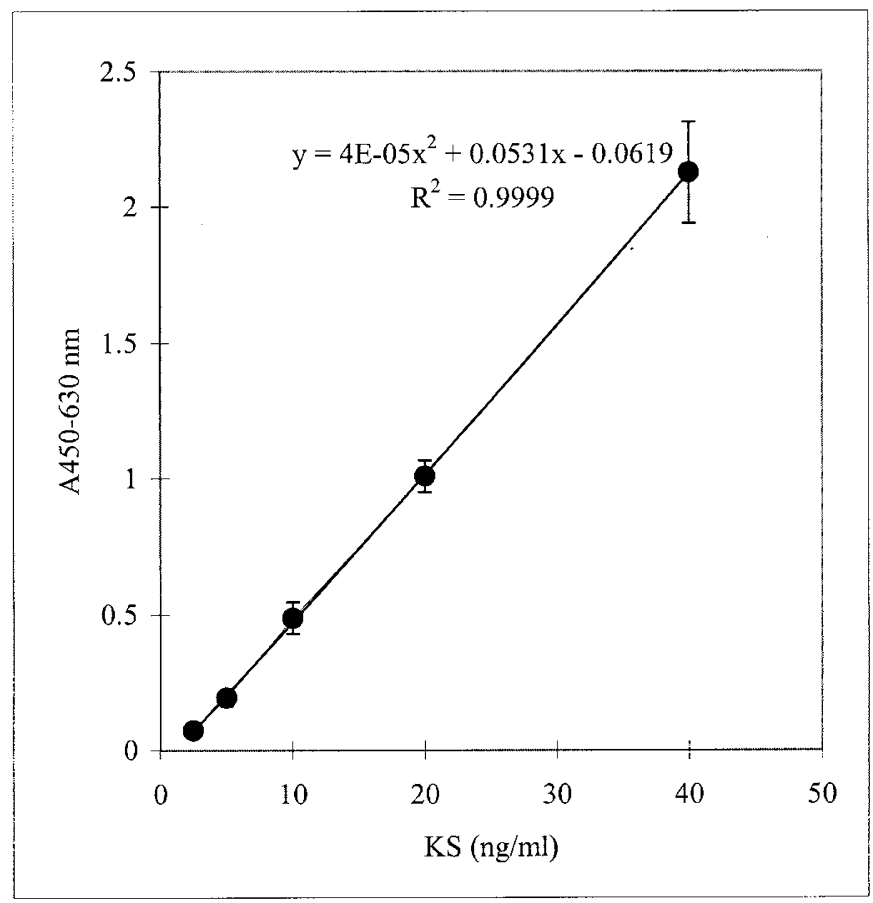

Figure 1. Standard curve for the KS ELISA. For validation, three different KS concentration quality control samples were prepared. The absorbances (minus blank) were plotted using $\mathrm{KS}(2.5,5.0,10.0,20.0,40.0 \mathrm{ng} / \mathrm{mL})$ standards.

$40.0 \mathrm{ng} / \mathrm{mL} \mathrm{KS}$ standards and quality controls was $<15 \%$. The inter-assay $\mathrm{CV}$ of three quality controls was calculated as $<10 \%$.

The specificity of the immunoassay for KS was assessed by determining the cross-reactivity (\%) to structurally related compounds from the AMPS kit. The 1000, 100, 10, and 2.5 $\mathrm{ng} / \mathrm{mL}$ solutions of the following compounds were analyzed in ELISA: CS-A, CS-C, DS, HS, and heparin. Cross-reactivity (\%) relative to the same concentration of KS standards was calculated and values were all $<0.1 \%$.

Recovery was assessed by adding the four different concentrations of KS standard to the plasma samples. The recovery (\%) of the added $\mathrm{KS}$ at $2.5,5,10$, and $20 \mathrm{ng} / \mathrm{mL}$ was all 100 $\pm 10 \%$. KS was stable for more than $6 \mathrm{y}$ in biologic samples stored at $-20^{\circ} \mathrm{C}$. Three cycles of freeze/thaw also had no effect on the KS levels.

Blood samples with KS concentrations ranging from 200 to $1500 \mathrm{ng} / \mathrm{mL}$ were assayed in triplicate on separate occasions using the appropriate dilution. The values for blood samples from 48 MPS IVA subjects with an identified age (mean age, $12.2 \mathrm{y}$; range, 0-65.6 y), 139 control subjects (mean age, $19.0 \mathrm{y} ; 0-80 \mathrm{y}$ ), and 37 cord blood samples are reported in Table 1.

Urine samples ranging from 0.05 to $40 \mathrm{mg} / \mathrm{g}$ creatinine were assayed in triplicate on separate occasions using the appropriate dilution. The results for urine samples from 70 MPS IVA subjects with an identified age (median age, $13.3 \mathrm{y} ; 0-65.6 \mathrm{y}$ ), and 154 control subjects (mean age, $9.3 \mathrm{y}$; range, $0-64 \mathrm{y}$ ) are reported in Table 2.

KS concentrations in the blood (Table 1) and urine (Table 2) are shown grouped by age or clinical severity. The blood and urine KS concentrations were found to vary markedly with age (Tables 1 and 2, Figs. 2 and 3). For the normal control individuals, during the first $5 \mathrm{y}$ of life, blood KS concentration rose progressively reaching a peak between age 5 and $10 \mathrm{y}$ (mean; $156 \mathrm{ng} / \mathrm{mL}$ for control, $776 \mathrm{ng} / \mathrm{mL}$ for MPS IVA patients; $p<0.0001$ ). After $12-13 \mathrm{y}$, blood KS concentrations decreased until age $20 \mathrm{y}$ and stabilized thereafter. When control subjects $(n=62)$ and MPS IVA patients $(n=12)$ over $20 \mathrm{y}$ of age were compared, the plasma or serum concentrations of KS were still significantly different between the groups (140 ng/mL versus $265 \mathrm{ng} / \mathrm{mL} ; p=0.015)$ (Table 1, Fig. 2). However, 6 out of 12 patients had blood KS levels within the normal range. After age 40, the blood KS levels in all three patients were within normal limits.

Urine KS levels for MPS IVA patients $(n=59)$ and normal controls $(n=95)$ were different at various ages (Fig. 3). During the first y of life, the mean concentrations of KS in the urine were $0.26 \mathrm{mg} / \mathrm{g}$ creatinine for the control group $(n=37)$ and $15.3 \mathrm{mg} / \mathrm{g}$ creatinine for MPS IVA patients $(n=10)(p<$ 0.0001 ). After age $4-5$, urine KS levels in MPS IVA began to decrease gradually. This decrease was most remarkable in the patient group between ages 5 and $10 \mathrm{y}(14 \mathrm{mg} / \mathrm{g}$ creatinine) and 10 and $15 \mathrm{y}(5.5 \mathrm{mg} / \mathrm{g}$ creatinine $)$. After age 15 , the $\mathrm{KS}$

Table 1. Blood KS (ng/mL) by ELISA method

\begin{tabular}{|c|c|c|c|c|c|c|}
\hline Age & Mean & $\mathrm{SD}$ & Maximum & Minimum & No. & Mean age \\
\hline Control (all ages) & 156 & 59.8 & 323 & 15 & 117 & 22.5 \\
\hline$>1, \leq 5 \mathrm{y}$ & 161 & 47.9 & 274 & 73 & 33 & 2.2 \\
\hline$>5, \leq 10 y$ & 234 & 76.9 & 323 & 80 & 10 & 6.5 \\
\hline$>10, \leq 15 y$ & 191 & 47.2 & 275 & 141 & 9 & 11.7 \\
\hline$>15, \leq 20 y$ & 77 & 21.7 & 91 & 52 & 3 & 18.7 \\
\hline$>20 y$ & 140 & 51.6 & 244 & 15 & 62 & 37.1 \\
\hline MPS IVA (all ages) & 525 & 330 & 1525 & 101 & 45 & 12.2 \\
\hline$>1, \leq 5 \mathrm{y}$ & 496 & 74.2 & 587 & 406 & 4 & 3 \\
\hline$>5, \leq 10 y$ & 776 & 336 & 1525 & 382 & 17 & 7.8 \\
\hline$>10, \leq 15 y$ & 589 & 235 & 899 & 326 & 6 & 11.6 \\
\hline$>15, \leq 20 y$ & 291 & 35.9 & 346 & 237 & 6 & 17.5 \\
\hline$>20 \mathrm{y}$ & 266 & 200 & 714 & 101 & 12 & 33.4 \\
\hline All severe & 561 & 344 & 1525 & 103 & 36 & 14.3 \\
\hline All milder & 386 & 226 & 785 & 101 & 9 & 22.6 \\
\hline
\end{tabular}


Table 2. Urine KS (ng/g creatinine) by ELISA method

\begin{tabular}{|c|c|c|c|c|c|c|}
\hline Age & Mean & SD & Maximum & Minimum & No. & Average age \\
\hline Control (all ages) & 0.21 & 0.15 & 0.67 & 0.03 & 95 & 15 \\
\hline$>1, \leq 5 \mathrm{y}$ & 0.26 & 0.122 & 0.668 & 0.079 & 37 & 1.1 \\
\hline$>5, \leq 10 y$ & 0.2 & 0.134 & 0.49 & 0.068 & 12 & 6 \\
\hline$>15, \leq 20 y$ & 0.14 & 0.046 & 0.194 & 0.08 & 6 & 16.1 \\
\hline$>20 \mathrm{y}$ & 0.12 & 0.093 & 0.418 & 0.029 & 26 & 36.3 \\
\hline MPS IVA (all ages) & 9.27 & 9.5 & 46.2 & 0.114 & 59 & 14.2 \\
\hline$>10, \leq 15 y$ & 5.5 & 5.04 & 13.9 & 1.12 & 7 & 12 \\
\hline$>15, \leq 20 y$ & 2.66 & 1.77 & 6.16 & 0.616 & 8 & 17.5 \\
\hline$>20 y$ & 2.23 & 1.51 & 4.7 & 0.114 & 12 & 35.5 \\
\hline Severe & 11.21 & 9.72 & 46.2 & 0.56 & 47 & 11.6 \\
\hline Milder & 1.67 & 1.43 & 4.42 & 0.114 & 12 & 24.3 \\
\hline
\end{tabular}

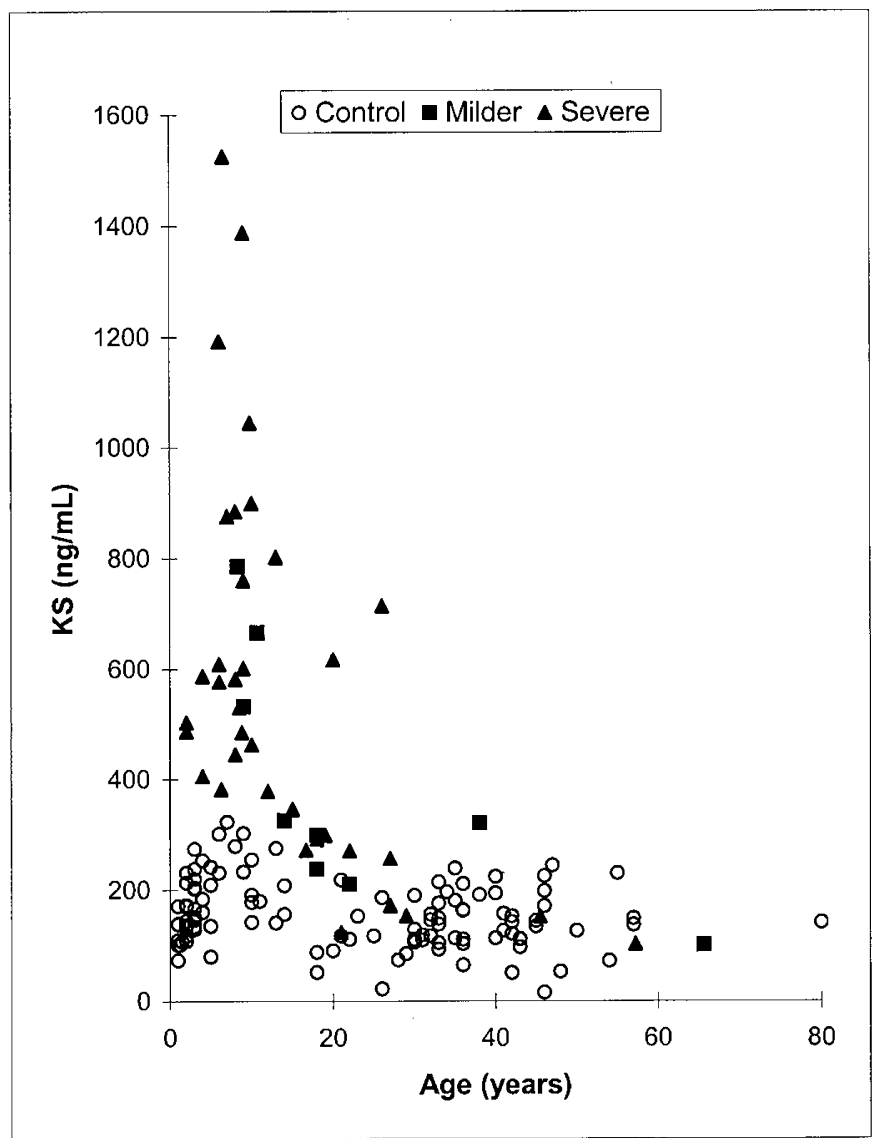

Figure 2. Concentrations of blood KS of patients with MPS IVA and normal individuals. Results of 45 specimens from MPS IVA individuals and 117 from normal individuals are plotted with respect to age. The blood KS reached a peak in the 5- to 10-y-old group in both patients and controls.

excretion continued to decline and reached a plateau after $20 \mathrm{y}$. The excretion of urine KS was significantly different between MPS IVA patients $(n=12)$ and normal controls $(n=26)$ after age 20 y $(2.23 \mathrm{mg} / \mathrm{g}$ creatinine versus $0.12 \mathrm{mg} / \mathrm{g}$ creatinine; $p$ $<0.0001$ ) (Table 2, Fig. 3). Although the degree of decline in the urine KS concentration proportional to age paralleled that of blood KS concentration, urine KS level remained higher than the control(Fig. 3).

In contrast to urine KS excretion, urine GAG excretion did not clearly distinguish the MPS IVA patients from normal

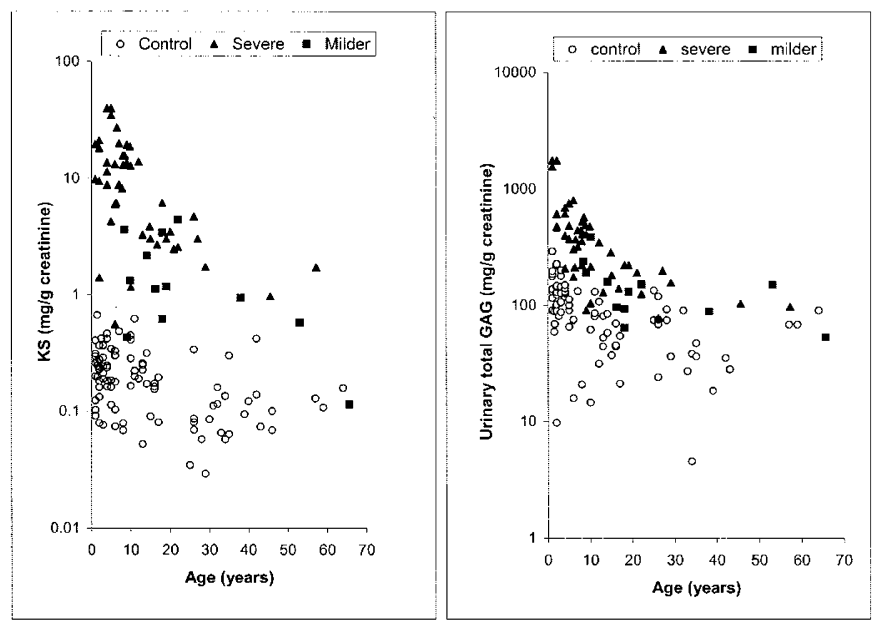

Figure 3. Relationship between urine KS or total GAG and age in MPS IVA patients and controls. (Left) Urine KS of patients with MPS IVA and normal individuals. Results of 59 specimens from MPS IVA patients and of 95 from normal individuals were plotted on a semilogarithmic scale with respect to age. (Right) GAG excretion of MPS IVA patients and normal individuals. Results of 59 specimens from MPS IVA patients and of 95 from normal individuals were plotted on a semilogarithmic scale with respect to age.

individuals (Fig. 3). Approximately 20\% of MPS IVA patients overlapped with the normal range. Urine KS and GAG concentrations were assayed in simultaneous samples, and there was a statistically significant correlation coefficient $(r=0.452$, $p=0.0004$ ) between urine KS and GAG in MPS IVA patients (Fig. 4). However, there was great variability with several outliers.

There was also a significant relationship between clinical severity and urine KS excretion. Patients with a severe form ( $n$ = 47) showed $11.2 \mathrm{mg} / \mathrm{g}$ creatinine on average, whereas the patients with a milder form had $1.67 \mathrm{mg} / \mathrm{g}$ creatinine $(n=12)$ $(p<0.0001$, Table 2$)$. The relationship between clinical severity and blood KS concentration was similar in that severe MPS IVA patients $(n=36)$ had 1.5 times higher KS concentrations than milder ones $(n=9)$ although this was statistically insignificant probably due to the small sample size $(p=0.169)$.

\section{DISCUSSION}

The accumulation of undegraded KS leads to damage of cartilage proteoglycans causing systematic skeletal dysplasia in 

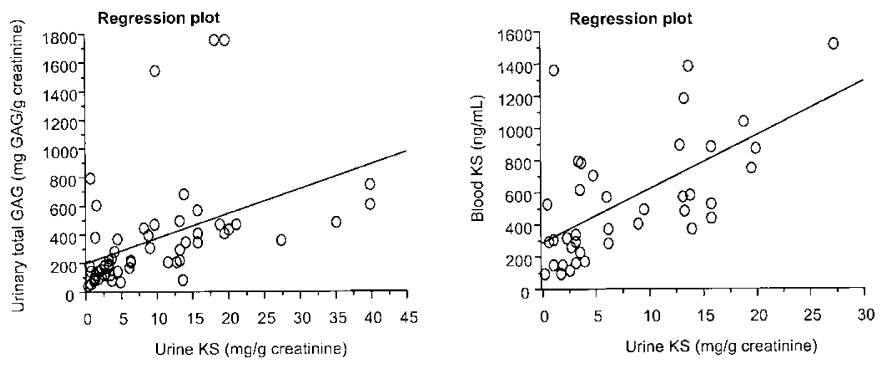

Figure 4. Correlation between urine $\mathrm{KS}$ and total GAG or blood KS in MPS IVA $(n=58)$. (Left) Correlation between urine KS and total GAG in MPS IVA-affected samples $(n=58)$. Linear regression analysis of these data yielded the equation $y=203.152+17.117 x$, where $x$ and $y$ are the concentrations of urine KS and total GAG, respectively. Person's correlation coefficient was 0.452 ( $p=0.0004)$. (Right) Correlation between blood and urine KS from MPS IVA-affected samples $(n=41)$. Person's correlation coefficient was 0 . Linear regression analysis of these data yielded the equation $y=284.022+$ $33.487 x$, where $x$ and $y$ are the concentrations of urine $\mathrm{KS}$ and blood KS, respectively.

MPS IVA patients. KS, which contributes more than $25 \%$ of the cartilage GAG in adults, is one of the most important components of bone. The degraded products of KS diffuse rapidly out of the cartilage matrix (9). When cartilage proteoglycans, like KS, are not degraded properly, they are stored mainly in chondrocytes. Although pathohistological examinations of the bone and cartilage cells are useful for diagnosing and staging MPS IVA patients, it is not convenient to obtain biopsy samples from MPS IVA patients. Measurements of KS concentrations in blood and urine samples should provide alternative information about the clinical status of MPS IVA patients.

The anti-KS MAb (5-D-4) adopted in this study has been used to quantify $\mathrm{KS}$ in blood of a normal population and in patients with arthritis (10-12). It had never been applied to MPS IVA patients previously. The 5-D-4 KS antibody is known to be specific for the portion of the KS molecule containing a sequence of three or more repeating units of disaccharide, sulfated $N$-acetyl-glucosamine-sulfated galactose (13). This epitope has not been found in any other GAG nor in proteoglycans. In our study, the absence of cross reactivity with other GAG confirms the specificity of the anti-KS antibody.

We developed a rapid, sensitive ELISA for measuring KS in blood and urine samples. In this and earlier studies (4), urine total GAG concentrations in MPS IVA patients measured by the DMB assay were similar to those in normal control samples. In contrast, our ELISA for KS in blood and urine distinguished MPS IVA patients from the controls with the exception of a few cases. The previous reports described that in adult or mild forms of MPS IVA, patients had no keratosulfaturia by the cetylpyridinium chloride method followed by thin-layer chromatography $(14,15)$. Using the ELISA method, the incidence of nonkeratosulfaturic MPS IVA would probably be lower than reported previously. A disproportionate increase in total urine GAG compared with urine KS was seen in some patients (Fig. 4), indicating that non-KS GAG species are excreted in the urine of these patients in large amounts. These patients were all between 1 and $4 \mathrm{y}$ of age and would be expected to have a high cartilage turnover resulting in high total GAG excretion. The higher level of non-KS species in their urine may represent $\mathrm{C6S}$, because it is known that abnormally high amounts of C6S are stored in MPS IVA patients. However, clarification of the higher levels of non-KS excretion needs further investigation.

Age-dependent changes in KS turnover (10) showed that blood KS level rose progressively during the first $4 \mathrm{y}$ of life, remained elevated until $12 \mathrm{y}$ of age, and then declined after age 13 until it stabilized at age 20. Elongation of the long bones during growth occurs through a process of endochondral ossification in which new cartilage is continuously laid down before it is degraded and replaced by bone. The decreased level of KS level after age 13 is consistent with the fact that the growth rate in normal children begins to decline after age 13 . These maturation-related changes are supported by the present study on MPS IVA. The new finding that the KS level in cord blood samples was lower than any other age group may indicate low metabolic activities in growing cartilage at birth or efficient placental clearing of GAG. After age 30, three out of four MPS IVA patients had normal blood KS levels and the extent of elevation of urine KS was reduced, suggesting that elevation of KS in MPS IVA is directly related to the rate of cartilage catabolism.

Maintaining an elevated urine KS concentration, in spite of normalization of blood KS concentration in adult MPS IVA patients (Fig. 3), raises another interesting question regarding KS metabolism. A lower rate of catabolism of proteoglycans in adult cartilage and/or decrease of mass of cartilage per body weight causes primary reduction of blood and urine KS concentration. Young MPS IVA patients have progressive destruction of cartilage tissues, releasing large quantities of KS at the initial stage. When the growth plate is closed or torn, synthesis of KS in cartilage will decline. To understand this phenomenon of normalization of blood KS concentration in adult MPS IVA patients, blood and urine KS levels need to be determined sequentially at different ages in the same individuals.

Patients with high levels of KS in the blood and urine likely represent severe cases of MPS IVA whose cartilage is overloaded with undegraded KS. In the progressive stage between ages 5 and 10, the mean blood KS concentration in MPS IVA patients was the highest. These findings indicate that the blood and urine KS concentrations in MPS IVA patients directly reflect the amount of stored KS in cartilage tissues.

\section{CONCLUSION}

In summary, determination of blood and urine KS concentrations by the ELISA method should provide a useful tool to assess clinical status in MPS IVA patients and measure response to treatments such as enzyme replacement therapy, bone marrow transplantation, and gene therapy.

Acknowledgments. The authors thank Dr. William S. Sly for constructive criticism and support. We also thank Drs. Robert W. Wilmott and Gary S. Gottesman for their review of this manuscript. 


\section{REFERENCES}

1. Neufeld EF, Muenzer J 2001 The mucopolysaccharidoses. In: Scriver CR, Beaudet AL, Sly WS, Valle D (eds) The Metabolic and Molecular Bases of Inherited Disease, 8th Ed. McGraw-Hill, New York, pp 3421-3452

2. Northover H, Cowie RA, Wraith JE 1996 Mucopolysaccharidosis type IVA (Morquio syndrome): a clinical review. J Inherit Metab Dis 19:357-365

3. Fang-Kircher SG, Herkner K, Windhager R, Lubec G 1997 The effects of acid glycosaminoglycans on neonatal calvarian cultures - a role of keratan sulfate in Morquio syndrome? Life Sci 61:771-775

4. Whitley CB, Ridnour MD, Draper KA, Dutton CM, Neglia JP 1989 Diagnostic tes for mucopolysaccharidosis I. Direct method for quantifying excessive urinary glycosaminoglycan excretion. Clin Chem 35:374-379

5. Bjornsson S 1993 Simultaneous preparation and quantitation of proteoglycans by precipitation with alcian blue. Anal Biochem 210:282-291

6. Whitley CB, Spielmann RC, Herro G, Teragawa SS 2002 Urinary glycosaminoglycan excretion quantified by an automated semimicro method in specimens conveniently transported from around the globe. Mol Genet Metab 75:56-64

7. Thonar EJ, Lenz ME, Klintworth GK, Caterson B, Pachman LM, Glickman P, Katz R, Huff J, Kuettner KE 1985 Quantification of keratan sulfate in blood as a marker of cartilage catabolism. Arthritis Rheum 28:1367-1376
8. Caterson B, Christner JE, Baker JR 1983 Identification of a monoclonal antibody that specifically recognizes corneal and skeletal keratan sulfate. Monoclonal antibodies to cartilage proteoglycan. J Biol Chem 258:8848-8854

9. Kimura JH, Osdoby P, Caplan AI, Hascall VC 1978 Electron microscopic and biochemical studies of proteoglycan polydispersity in chick limb bud chondrocyte cultures. J Biol Chem 253:4721-4729

10. Thonar EJ, Pachman LM, Lenz ME, Hayford J, Lynch P, Kuettner KE 1988 Age related changes in the concentration of serum keratan sulphate in children. J Clin Chem Clin Biochem 26:57-63

11. Sweet MB, Coelho A, Schnitzler CM, Schnitzer TJ, Lenz ME, Jakim I, Kuettner KE, Thonar EJ 1988 Serum keratan sulfate levels in osteoarthritis patients. Arthritis Rheum 31:648-652

12. Thonar EJ, Manicourt DM, Williams J, Lenz ME, Sweet MB, Schnitzer TJ, Otten L, Glant T, Kuettner KE 1991 Circulating keratan sulfate: a marker of cartilage proteoglycan catabolism in osteoarthritis. J Rheumatol Suppl 27:24-26

13. Mehmet H, Scudder P, Tang PW, Hounsell EF, Caterson B, Feizi T 1986 The antigenic determinants recognized by three monoclonal antibodies to keratan sulphate involve sulphated hepta- or larger oligosaccharides of the poly ( $N$-acetyllactosamine) series. Eur J Biochem 157:385-391

14. Fujimoto A, Horwitz AL 1983 Biochemical defect of non-keratan-sulfate-excreting Morquio syndrome. Am J Med Genet 15:265-273

15. Beck M, Glossl J, Grubisic A, Spranger J 1986 Heterogeneity of Morquio disease. Clin Genet 29:325-331 